\title{
Performance Reliability Prediction of Complex System Based on the Condition Monitoring Information
}

\author{
Hongxing Wang ${ }^{1}$ and Yu Jiang ${ }^{2}$ \\ ${ }^{1}$ Jiangsu Frontier Electric Technology Co., Ltd., Nanjing 211102, China \\ ${ }^{2}$ College of Civil Aviation, Nanjing University of Aeronautics and Astronautics, Nanjing 20016, China \\ Correspondence should be addressed to Yu Jiang; jiangyu07@nuaa.edu.cn
}

Received 18 June 2013; Revised 23 September 2013; Accepted 23 September 2013

Academic Editor: Hamid Reza Karimi

Copyright (C) 2013 H. Wang and Y. Jiang. This is an open access article distributed under the Creative Commons Attribution License, which permits unrestricted use, distribution, and reproduction in any medium, provided the original work is properly cited.

\begin{abstract}
Complex system performance reliability prediction is one of the means to understand complex systems reliability level, make maintenance decision, and guarantee the safety of operation. By the use of complex system condition monitoring information and condition monitoring information based on support vector machine, the paper aims to provide an evaluation of the degradation of complex system performance. With degradation assessment results as input variables, the prediction model of reliability is established in Winer random process. Taking the aircraft engine as an example, the effectiveness of the proposed method is verified in the paper.
\end{abstract}

\section{Introduction}

The reliability of complex systems is directly related to operation safety, so the reliability prediction is of great importance. Different from the method based on the reliability of fault prediction, complex systems mainly use condition monitoring parameter to predict the complex system performance degradation state. When the threshold of the degradation state goes beyond the prescribed one, timely maintenance and inspection are being done to avoid accidents. Therefore, the reliability of complex systems is mainly aimed at the reliability of the performance degradation prediction.

Complex system reliability prediction has received extensive attention of many scholars. One trend in the research about this problem is using different temporal point data to predict the future level of reliability based on the analysis of the data change trend. Lu et al. [1] presented an evaluation model of real-time performance based on time series method and researched the reliability prediction of the bit excessive wear failure by regarding drill thrust as performance monitoring parameters; Elwany and Gebraeel [2] presented a model for predicting system performance reliability based on Bayesian, and applied to parts replacement and inventory decisions; Li and Masuda [3] discussed the multistate coherent system composed of multistate components. In view of degradation signal monitor parts reliability, Chinnam [4] made use of the reliability condition of some parts which performance degenerate signals were monitored and adopted a general polynomial regression model to describe performance change. In addition, based on the analysis of the relationship between state parameters, the performance change rules on study was put forward, which can be used to predict the reliability level at a particular moment or under specified reliability level of residual life. Condition monitoring is the key to access the operational reliability. Christer et al. [5] used a state space model to predict the erosion condition of the inductors in an induction furnace in which a measure of the conductance ratio (CR) is used to indirectly assess the relative condition of the inductors and to guide replacement decisions. Bharadwaj and Parlos [6] developed a sensorless neural adaptive speed filter for induction motors operating under normal conditions and running off the power supply mains. Chen et al. [7] proposed an integrated RUL prediction method using adaptive neuro-fuzzy inference systems (ANFIS) and high-order particle filtering, which forecast the time evolution of the fault indicator and estimates the probability density function (pdf) of RUL.

Bosnić and Kononenko [8] compared different approaches to estimate the reliability of individual predictions in 
regression and to compose a combined estimate that performs better than the individual estimates; Wang and Coit [9] made an analysis of the complex relationship between monitoring variables and proposed a prediction method of correlation between variables with multifunctional parameters and verified the effectiveness of the proposed method by simulation data; $\mathrm{Xu}$ et al. [10] established the model of real-time reliability evaluation based on performance degradation by assuming that degradation function conformed to the Brownian motion and drift; $\mathrm{Lu}$ et al. [11] presented a technique for predicting system performance reliability in real-time considering multiple failure modes. The technique includes on-line multivariate monitoring and forecasting of selected performance measures and conditional performance reliability estimates based on collaborative filtering; Xu et al. [12] introduced a new real-time reliability prediction method for dynamic systems; Hernando et al. [13] introduced the idea of using a reliability measure associated with the predictions made by recommender systems; in order to improve data utilization results, $\mathrm{Li}$ et al. [14] proposed a method based on grey model to predict the reliability and verified the validity of the model by applying it in the manufacturing system data of electronic product; $\mathrm{Hu}$ et al. [15] adopted the method of D-S evidence for scientific forecast and verified its validity by its application to engine turbosupercharging system; Moura et al. [16] conducted an analysis of the different sequential points data and made a reliability prediction by the use of support vector machine (SVM) method; Lolas and Olatunbosun [17] presented the first module of an expert system, a neural network architecture that could predict the reliability performance of a vehicle at later stage of its life by using only information from first inspection after the vehicle's prototype production; $\mathrm{Hu}$ et al. [18] developed a novel reliability prediction technique based on the evidential reasoning (ER) algorithm which was applied to forecast reliability in turbocharger engine systems. And the experimental results showed that the prediction performance of the ER-based prediction model outperformed several existing methods in terms of prediction accuracy or speed.

The above research is mainly aimed to realize the goal of reliability prediction by using the methods of artificial intelligence and information fusion methods to train and study the data. Due to the complicated system with abundant condition monitoring parameters and small sample system, the paper put forward a method to evaluate complex system performance degeneration by adopting information fusion methods and described the performance degeneration of complex system under random process condition. The main aim of the paper is to realize the goal of improving reliability of forecast accuracy and credibility.

The research results in the paper can be further used in the following areas.

(1) The research results can be applied to control risk in complex system operation. By means of the real-time monitoring and tracking, the major accidents can be avoided.
(2) The research results can be applied to health management field of complex system. The assessment of operational reliability is the core in health management. Improving the accuracy of assessment in operation reliability can increase the operational efficient in healthy management.

(3) The research results can afford supports for the maintenance decision. The complex system is typical repairable system. The maintenance decision is not only related to operational safety but also operational cost. It is necessary to enhance the accuracy of maintenance decision, because that decision basis in maintenance is the reliability standards in complex system.

(4) Improving the value of condition monitoring information can provide new thoughts in design process. In order to ensure the safety and reliability of the operation system and consistently high-quality products, it is still necessary to develop an efficient process monitoring scheme to detect the abnormal situations as early as possible and take corrective actions in time.

\section{Framework of the Complex System Reliability Prediction}

Complex systems are characterized with abundant condition monitoring parameters and small sample system. Considering these characteristics, support vector machine (SVM) method is used in the research because of its unique advantages in dealing with a small sample data.

Support vector machine (SVM) is a learning system proposed by Vapnik. SVM is put forward according to the structural risk minimization principle in statistical learning theory and uses linear function hypothesis space in highdimensional feature space. SVM is used to seek the best compromise between complexity and learning ability according to the limited sample information of the model. Because of the forecast superiority in the treatment of small sample, SVM realizes efficient transmission reasoning from training sample to forecast sample. It can reasonably solve the actual problems such as small sample, nonlinear, high dimension, and local minimum point.

Applying SVM method, comprehensive utilization of multisource monitoring parameters and assessment of performance degradation in complex systems can be completed. Because performance retirement leading to reliability is a random course, the paper establishes reliability prediction model for dynamic assessment of the complex systems reliability by choosing Winer process complex system performance degradation.

The process of reliability prediction of complex systems is shown in Figure 1.

The advantages of algorithm in the paper include some aspects.

(1) The algorithm can deal with the prediction of realtimereliability sufficiently in complex system in view 


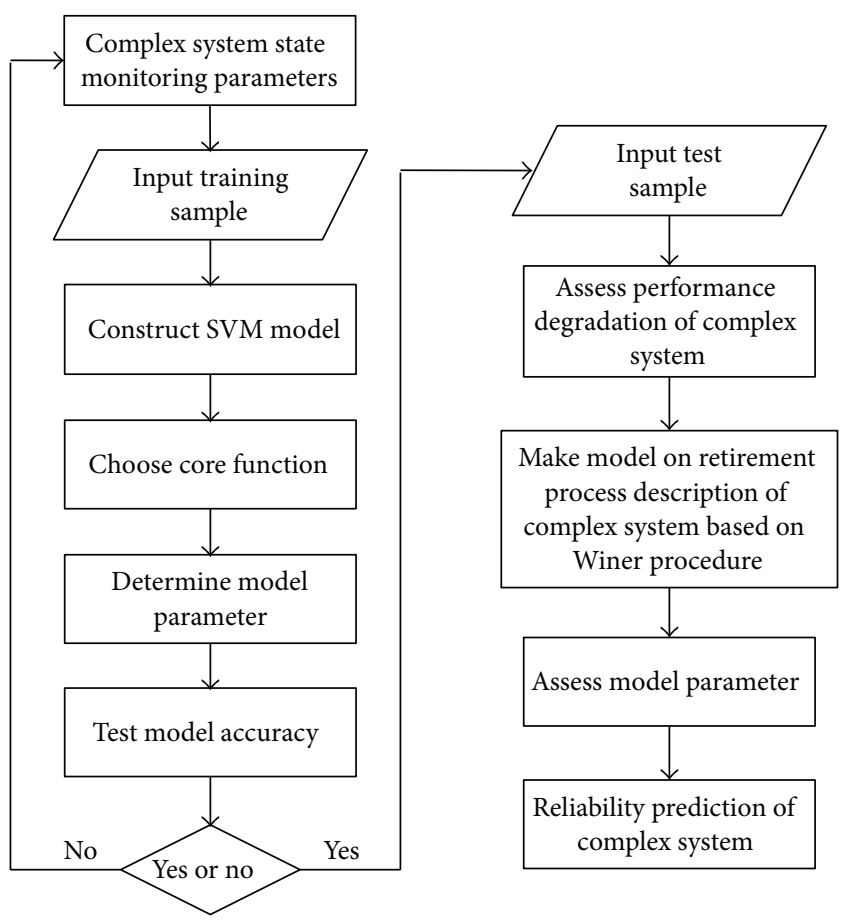

FIGURE 1: Performance degradation assessment of complex system.

of small sample and solve the question efficiently which is difficult to collect mass data because of the high cost.

(2) The algorithm can exploit multisource monitoring information comprehensively to improve the accuracy of condition monitoring.

(3) The algorithm can indicate the reliable change rules which are lead by the random disturbance of multiple factors, and it confirms to the actual operation of complex system.

(4) The algorithm can analyze the influence on operational reliability accurately produced by condition change. Therefore, it can control the risk efficiently.

\section{Performance Degradation Assessment Model of Complex System Based on SVM}

Assuming sample training set is $\left\{\left(x_{i}, y_{i}\right) \mid i=1,2, \ldots, l\right\}$, $x_{i} \in R^{n}$ refers to the input variables, which corresponds to condition monitoring parameters of complex system; $y_{i} \in R^{n}$ refers to the output variables. Generally, vector machine can use nonlinear mapping $\varphi(\cdot)$ to get a high-dimensional feature space from the original monitoring parameters, where it can construct the optimal decision function: $f(x)=(w \cdot \varphi(x))+b$, where $w \cdot \varphi(x)$ means dot-product of vectors $w$ and mapping function. $b$ refers to bias. So the corresponding constraint optimization problem can be expressed as follows:

$$
\begin{aligned}
& \min _{w, b, \xi, \xi^{*}} \quad \frac{1}{2}\|w\|^{2} \frac{1}{2}+C \sum_{i=1}^{i}\left(\xi_{i}+\xi_{i}^{*}\right) \\
& \text { s.t. } \quad\left\{\begin{array}{l}
y_{i}-w^{T} \cdot \psi\left(x_{i}\right)-b \leq \varepsilon+\xi_{i} \\
w^{T} \cdot \psi\left(x_{i}\right)+b-y_{i} \leq \varepsilon+\psi_{i}^{*} \\
\xi_{i}, \xi_{i}^{*} \geq 0
\end{array}\right. \\
& i=1,2, \ldots, l \text {. }
\end{aligned}
$$

In formula (1), $C$ is the penalty factor, which implements the empirical risk and confidence range of a compromise, $\xi_{i}$, $\xi_{i}^{*}$ refer to slack variables, which, respectively, represent the ceiling and floor of training error in the epsilon zero error constraints $\left(\left|y_{i}-\left[w^{T} \cdot \psi\left(x_{i}\right)+b\right]\right|<\varepsilon\right) ; \varepsilon$ refers to the error defined by insensitive cost function Vapnik- $\varepsilon$. The optimization problem determined by formula (1) is a typical convex quadratic programming one. According to Lagrange theory, weight vector $w$ equals a linear combination of the training data.

$$
w=\sum_{i=1}^{i}\left(\alpha_{i}-\alpha_{i}^{*}\right) \psi\left(x_{i}\right) .
$$

Substituting formula (2) into (1), we obtain predictive value of unknown point $x$. It can be expressed as follows:

$$
f(x)=\sum_{i=1}^{l}\left(\alpha_{i}-\alpha_{i}^{*}\right) K\left(x_{i}, x\right)+b .
$$

In (3), $K\left(x_{i}, x\right)=\psi\left(x_{i}\right) \cdot \psi(x)$ is known as the kernel function.

According to the above optimization problem, the key is the choice of kernel function. There is no general method or theory to select the optimal kernel function so far, so modeling is necessary in the practice to choose a different kernel function for specific objects based on the past experience and simulation. At present more than 10 kinds of kernel function are used. In view of the nature of the question investigated in this paper, the paper chooses Radial Basis Function (RBF) as the kernel function, whose expression is described as follows:

$$
K\left(x, x_{i}\right)=\exp \left(\frac{\left\|x-x_{i}\right\|^{2}}{\delta^{2}}\right) .
$$

\section{Reliability Prediction of Complex System Based on Wiener Process}

Complex system performance degradation is a stochastic process. The paper chooses the Wiener process to describe the process of complex system degradation, denoted by $w(t)$ as follows:

$$
w(t)=\eta t+\delta B(t), \quad t \geq 0
$$


The stochastic process is defined as $\{W(t)\}$. If $t>0$, $\{W(t)\}$ is defined as Wiener process and satisfies the following assumptions:

(i) $w(0)=0$;

(ii) $\{w(t)\} ; t>0$ with stationary independent increments;

(iii) for any $t>0,\{w(t)\}$ is normal random variable, whose mean is 0 , and the variance is $\delta^{2} t$.

For any $0 \leq s<\infty,[W(t)-W(s)]$ follow Gaussian distributions $N\left[\eta(t-s), \delta^{2}(t-s)\right]$.

Assuming that the complex system failure threshold is $w$ the failure time of complex system is described as follows:

$$
T=\inf \{t ; w(t)>w\} .
$$

The distribution of $T$ is

$$
\begin{array}{r}
F_{T}(t)=\phi\left(\frac{\eta}{\delta} \sqrt{t}-\frac{w}{\delta \sqrt{t}}\right)+\phi\left(-\frac{\eta}{\delta} \sqrt{t}-\frac{w}{\delta \sqrt{t}}\right) \cdot e^{2 \eta w / \delta^{2}} \\
t>0
\end{array}
$$

Accordingly, at this time the complex systems performance reliability is:

$$
\begin{array}{r}
R(t)=1-\phi\left(\frac{\eta}{\delta} \sqrt{t}-\frac{w}{\delta \sqrt{t}}\right)-\phi\left(-\frac{\eta}{\delta} \sqrt{t}-\frac{w}{\delta \sqrt{t}}\right) \cdot e^{2 \eta w / \delta^{2}}, \\
t>0 .
\end{array}
$$

The parameters $u$ and $\beta$ are described as follows:

$$
\begin{gathered}
u=\frac{w}{\eta}, \quad \beta=\frac{\delta^{2}}{\eta^{2}}, \quad u>0, \beta>0 \\
F_{T}(t)=\Phi\left(\frac{\sqrt{t}}{\sqrt{\beta}}-\frac{u}{\sqrt{\beta} \sqrt{t}}\right)+\Phi\left(-\frac{\sqrt{t}}{\sqrt{\beta}}-\frac{u}{\sqrt{\beta} \sqrt{t}}\right) \cdot e^{2 u / \beta} \\
t>0, u>0, \beta>0 .
\end{gathered}
$$

The probability distribution function is described as follows:

$$
f_{T}(t)=\frac{u}{\sqrt{2 \pi \beta t^{3}}} e^{-\left((t-u)^{2} / 2 \beta t\right)}, \quad t>0, u>0, \beta>0 .
$$

Distribution form for the above is Inverse Gaussian, denoted as $t \sim \operatorname{IG}(\mu, \beta)$.

Assuming that $\left(t_{1}, t_{2}, \ldots, t_{n}\right)$ is the performance degradation samples to be observed, defining $\Delta x$ as performance degradation of different time, then do the likelihood function of the probability density function of complex system life distribution, and the expression is shown as follows (12):

$$
\begin{aligned}
L(x, u, \beta) & =\prod_{i=1}^{n} f\left(x_{i} ; u, \beta\right) \\
& =\frac{u^{n}}{(2 \pi \beta)^{n / 2} \prod_{i=1}^{n}\left(x_{i}\right)^{3 / 2}} e^{\left\{-(1 / 2 \beta) \sum_{i=1}^{n}\left(\left(x_{i}-\mu\right)^{2} / x_{i}\right)\right\}} .
\end{aligned}
$$

Evaluate partial derivative of $\mu, \beta$ and obtain likelihood equations:

$$
\begin{aligned}
& \frac{\partial \ln L\left(x_{i} ; \mu, \beta\right)}{\partial \mu}=\frac{n}{\mu}+\frac{1}{\beta} \sum_{i=1}^{n} \frac{x_{i}-\mu}{x_{i}}=0, \\
& \frac{\partial \ln L\left(x_{i} ; \mu, \beta\right)}{\partial \beta}=\frac{n}{2 \beta}+\frac{1}{2 \beta^{2}} \sum_{i=1}^{n} \frac{\left(x_{i}-\mu\right)^{2}}{x_{i}}=0 .
\end{aligned}
$$

To solve the likelihood equations, we can get

$$
\begin{gathered}
\mu=\frac{1}{n} \sum_{i=1}^{n} x_{i}, \\
\beta=\left(\frac{1}{n} \sum_{i=1}^{n} x_{i}\right)^{2}\left(\frac{1}{n} \sum_{i=1}^{n} \frac{1}{x_{i}}\right)-\frac{1}{n} \sum_{i=1}^{n} x_{i} .
\end{gathered}
$$

\section{Case Study}

Taking aircraft engine as an example, the proposed algorithm is explained. The aircraft engine performance degradation (or efficiency) usually reflects the variation of the monitoring parameters. At present aircraft engine condition monitoring mainly includes the following content.

(1) Pneumatic performance monitoring: the civil aviation engine is the core component gas path system components. Some thermodynamic parameters can reflect the state of engine performance change. The monitoring parameters are turbine gas temperature (EGT) and fuel flow (WF).

(2) Oil lubricating monitoring: the objects of oil lubricating monitoring are lubrication system components and seal system. It is useful for mechanical wear fault monitoring and diagnosis. The monitoring parameters are oil pressure (OP), oil temperature (OT), and oil consumption rate (OCR) parameters, and so forth. The changes of the parameters are useful for engine condition monitoring.

(3) Vibration monitoring: because engine rotor wear or damage will produce a certain degree of vibration signal, mechanical damage of the engine can be observed by observing the vibration of the rotor and its components, including low pressure rotor vibration value deviation (ZVB1F) and the high pressure rotor vibration value deviation (ZVB2R).

EGT overweight, WF increase, and lager ZVB1F, ZVB2R, and OCR are all the indication of aircraft engine performance decline. These indicators can be thought of as internal covariate cause of aeroengine performance decline.

Table 1 is a sample of aircraft which needs to be changed. From the 20 samples data, it can get time since installation (TSI) and flight hour (FH). The paper uses the sample data as training sample to get the relation between performance degeneration degree and condition monitoring parameters.

In Table 2, the engine monitoring information is listed. Related parameter calculation and reliability prediction are 
TABLE 1: Key performance monitoring parameters for some aircraft engine.

\begin{tabular}{lcccccccc}
\hline Monitoring point & DEGT & GWFM & GPCN25 & DPOIL & ZVBIF & ZVB2R & TSI/FH & PDD \\
\hline 1 & 4.69 & 2.66 & 1.83 & -3.76 & 0.31 & 0.51 & 3282 & 0.0975 \\
2 & -0.63 & 4.91 & 2.00 & -5.74 & 0.43 & 0.76 & 3754 & 0.0459 \\
3 & -5.66 & 3.70 & 1.95 & -3.00 & 0.29 & 0.38 & 2640 & 0.1078 \\
4 & 5.25 & 4.06 & 2.00 & -5.55 & 0.89 & 0.65 & 4740 & 0.1176 \\
5 & -3.94 & 4.25 & 1.72 & -8.82 & 0.50 & 0.46 & 1707 & 0.1305 \\
$\vdots$ & $\vdots$ & $\vdots$ & $\vdots$ & $\vdots$ & $\vdots$ & $\vdots$ & $\vdots$ \\
16 & 17.41 & 6.03 & 2.08 & 9.26 & 0.29 & 0.33 & 6.988 \\
17 & 3.17 & 4.41 & 2.24 & 9.39 & 0.97 & 0.61 & 1477 \\
18 & -3.19 & 2.00 & 1.68 & 13.10 & 0.00 & 0.89 & 0.1725 \\
19 & 7.52 & 2.78 & 1.04 & -3.24 & 0.45 & 0.99 & 5021 & 0.1572 \\
20 & 4.43 & 3.77 & 2.30 & -5.96 & 0.52 & 0.48 & 0.1665 \\
\hline
\end{tabular}

TABLE 2: Performance degradation assessment and reliability prediction for aircraft engine.

\begin{tabular}{cccccccccccc}
\hline No. & & & & & & Item & & & & \\
& TSI $(\mathrm{h})$ & GWFM & GPCN25 & DPOIL & ZVBIF & ZVB2R & GWFM & DPP & DPP & error & $\widehat{R}$ \\
\hline 1 & 1422 & 5.2515 & 4.0629 & 2.0022 & 0.8911 & 0.6452 & 14.0458 & 0.0792 & 0.0826 & $4.29 \%$ & 0.9728 \\
2 & 1954 & 5.3187 & 4.2168 & 1.3963 & 0.0945 & 0.3609 & 14.9931 & 0.1129 & 0.1135 & $0.53 \%$ & 0.9563 \\
3 & 3330 & 17.4070 & 6.0285 & 2.0803 & 0.2878 & 0.3300 & 22.0301 & 0.1824 & 0.1761 & $-3.45 \%$ & 0.9195 \\
\hline
\end{tabular}

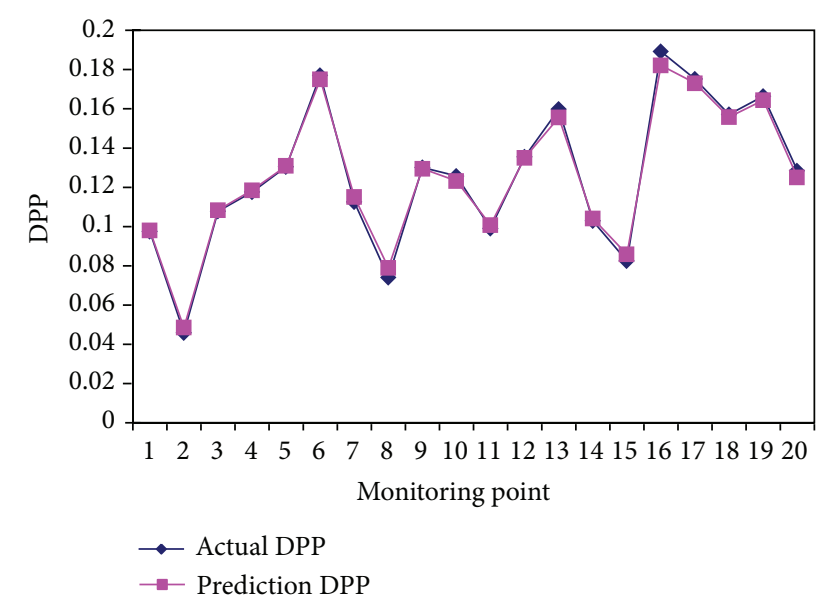

FIGURE 2: Training sample aircraft engine performance degradation of the actual value compared with forecast figure.

also listed in Table 2. Performance degradation degree is predicted by monitoring the relationship between parameters by 20 samples. The degree of performance degradation of the actual value (performance degradation degree, PDD) is not directly collected data but calculated by Monte-Carlo simulation method according to the engine under the wing of the remaining life and reliability at a given threshold (90\%) cases, when the performance degradation process conforms to the Winer stochastic process.

Using SVM method to extract the relationship between performance degradation and the monitoring parameters, the comparison between predicted values and actual values is shown in Figure 2.

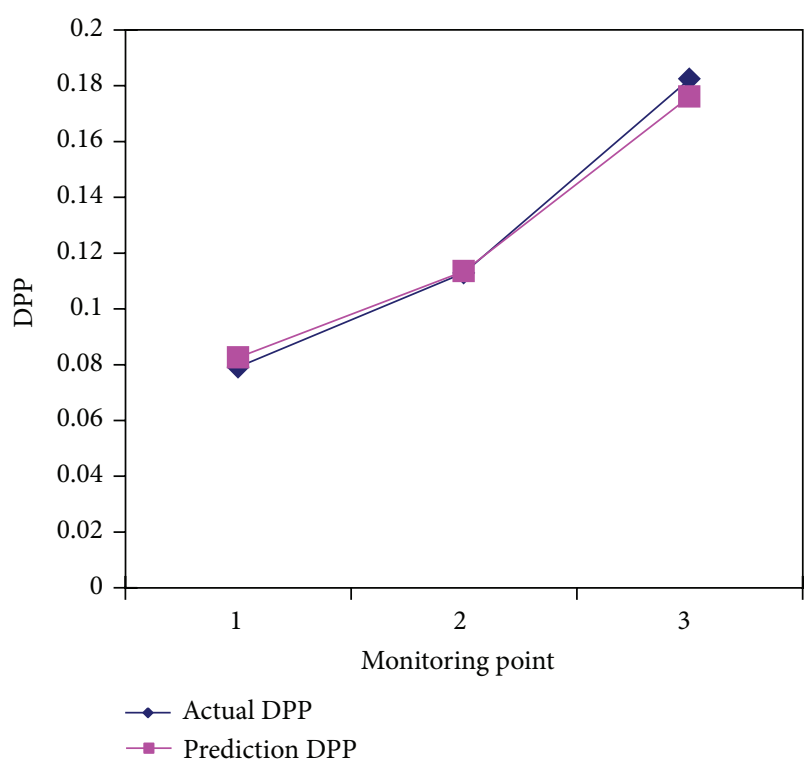

FIGURE 3: Real value and predictive value of a certain type of aircraft performance degradation.

Based on 20 training samples, the SVM method is used to get the relationship between monitoring parameters and the performance degradation, and the calculation results are shown in Table 2. And then compare actual performance degradation calculated by remaining life Backward Pass and the error which are shown in Table 2.

The contrast between real values and predicted values of performance degradation is shown in Figure 3. 


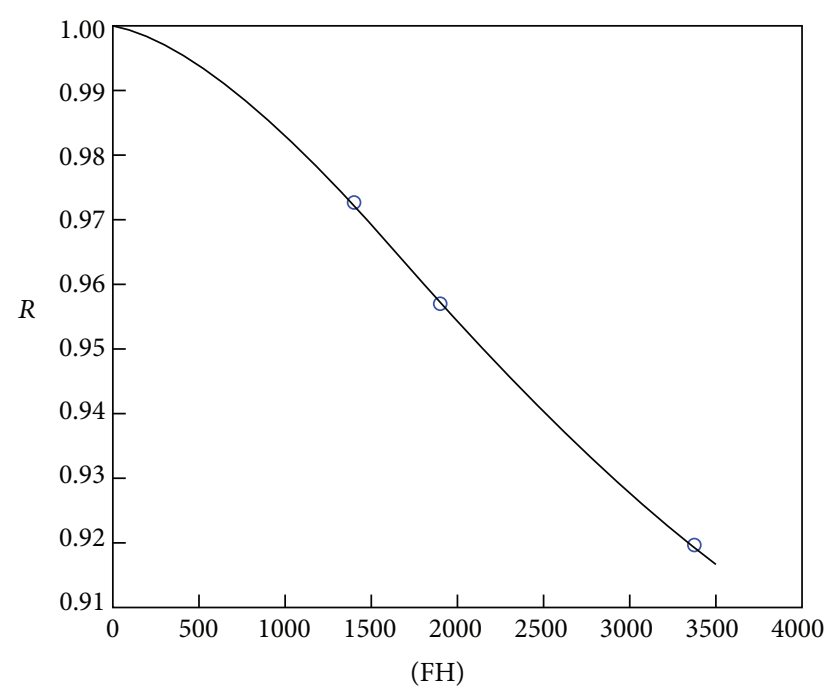

Figure 4: Performance reliability predictions for some aircraft engine.

By the formula (8), the future level of reliability can be predicted. Figure 4 indicates the performance reliability prediction curve of aeroengine. For example, if the cumulative time on wing (TSI) is 3600 flight hours, the result can be expressed as $\bar{R}(3600)=0.9167$.

The research focuses on exploit condition monitoring to access reliability in the paper at present. The further research will expand to operational reliability assessments of data driven in order to probe more comprehensive data utilization technologies and methods. The further research can expand to design field. The information on operational reliability assessment can be fed back to design process and analyze the influence. It is necessary to enhance the abstraction of related information for sensitive parameters in design process. On the contrary, the data collection demand of sensitive parameters can be reduced. Some scholars have already done some research on it. For example, Yin et al. [19] made a comparison research on basic data-driven methods for process monitoring and fault diagnosis. Yin et al. [20] presented two online schemes for an integrated design of fault-tolerant control systems with application to Tennessee benchmark.

In further, the research on condition monitoring and operational reliability of system integration will be developed.

\section{Conclusion}

By support vector machine (SVM) method and condition monitoring information, aviation engine performance degradation is evaluated. The result will be used as input variables of the reliability analysis model. Aircraft engine performance degradation process is described by the use of the Winer process in order to forecast aeroengine performance reliability using condition monitoring information. The method integrates the performance monitoring and reliability analysis into one framework, making full use of a variety of condition monitoring information, and so the result is more accurate.
Though much research has been done about this method, more studies are needed.

\section{Acknowledgments}

This work was supported by the National Natural Science Foundation of China and Civil Aviation Administration of China (no. U1333117), China Postdoctoral Science Foundation (no. 2012M511275), and The Basic Service Foundation of Nanjing University of Aeronautics and Astronautics (no. NS2013067).

\section{References}

[1] H. Lu, W. J. Kolarik, and S. S. Lu, "Real-time performance reliability prediction," IEEE Transactions on Reliability, vol. 50, no. 4, pp. 353-357, 2001.

[2] A. H. Elwany and N. Z. Gebraeel, "Sensor-driven prognostic models for equipment replacement and spare parts inventory," IIE Transactions, vol. 40, no. 7, pp. 629-639, 2008.

[3] J.-A. Li, Y. Wu, K. K. Lai, and K. Liu, "Reliability estimation and prediction of multi-state components and coherent systems," Reliability Engineering and System Safety, vol. 88, no. 1, pp. 9398, 2005.

[4] R. B. Chinnam, "On-line reliability estimation for individual components using statistical degradation signal models," Quality and Reliability Engineering International, vol. 18, no. 1, pp. 53-73, 2002.

[5] A. H. Christer, W. Wang, and J. M. Sharp, "A state space condition monitoring model for furnace erosion prediction and replacement," European Journal of Operational Research, vol. 101, no. 1, pp. 1-14, 1997.

[6] R. M. Bharadwaj and A. G. Parlos, "Neural state filtering for adaptive induction motor speed estimation," Mechanical Systems and Signal Processing, vol. 17, no. 5, pp. 903-924, 2003.

[7] C. Chen, G. Vachtsevanos, and M. E. Orchard, "Machine remaining useful life prediction: an integrated adaptive neurofuzzy and high-order particle filtering approach," Mechanical Systems and Signal Processing, vol. 28, pp. 597-607, 2012.

[8] Z. Bosnić and I. Kononenko, "Comparison of approaches for estimating reliability of individual regression predictions," Data and Knowledge Engineering, vol. 67, no. 3, pp. 504-516, 2008.

[9] P. Wang and D. W. Coit, "Reliability prediction based on degradation modeling $\mathrm{f}$ or system $\mathrm{s}$ with multiple deggradation measures," Reliability and Maintainability, vol. 78, no. 3, pp. 302-307, 2004.

[10] Z. Xu, Y. Ji, and D. Zhou, "Real-time reliability prediction for a dynamic system based on the hidden degradation process identification," IEEE Transactions on Reliability, vol. 57, no. 2, pp. 230-242, 2008.

[11] S. Lu, H. Lu, and W. J. Kolarik, "Multivariate performance reliability prediction in real-time," Reliability Engineering and System Safety, vol. 72, no. 1, pp. 39-45, 2001.

[12] Z. Xu, Y. Ji, and D. Zhou, "A new real-time reliability prediction method for dynamic systems based on on-line fault prediction," IEEE Transactions on Reliability, vol. 58, no. 3, pp. 523-538, 2009.

[13] A. Hernando, J. Bobadilla, F. Ortega, and J. Tejedor, "Incorporating reliability measurements into the predictions of a recommender system," Information Sciences, vol. 218, pp. 1-16, 2013. 
[14] G.-D. Li, S. Masuda, D. Yamaguchi, and M. Nagai, "A new reliability prediction model in manufacturing systems," IEEE Transactions on Reliability, vol. 59, no. 1, pp. 170-177, 2010.

[15] C.-H. Hu, X.-S. Si, and J.-B. Yang, "System reliability prediction model based on evidential reasoning algorithm with nonlinear optimization," Expert Systems with Applications, vol. 37, no. 3, pp. 2550-2562, 2010.

[16] M. D. C. Moura, E. Zio, I. D. Lins, and E. Droguett, "Failure and reliability prediction by support vector machines regression of time series data," Reliability Engineering and System Safety, vol. 96, no. 11, pp. 1527-1534, 2011.

[17] S. Lolas and O. A. Olatunbosun, "Prediction of vehicle reliability performance using artificial neural networks," Expert Systems with Applications, vol. 34, no. 4, pp. 2360-2369, 2008.

[18] C.-H. Hu, X.-S. Si, and J.-B. Yang, "System reliability prediction model based on evidential reasoning algorithm with nonlinear optimization," Expert Systems with Applications, vol. 37, no. 3, pp. 2550-2562, 2010.

[19] S. Yin, S. X. Ding, A. Haghani et al., "A comparison study of basic data-driven fault diagnosis and process monitoring methods on the benchmark Tennessee Eastman Process," Journal of Process Control, vol. 22, no. 9, pp. 1567-1581.

[20] S. Yin, H. Luo, and S. X. Ding, "Real time implementation of fault-tolerant control systems with performance optimization," IEEE Transactions on Industrial Electronics, 2013. 


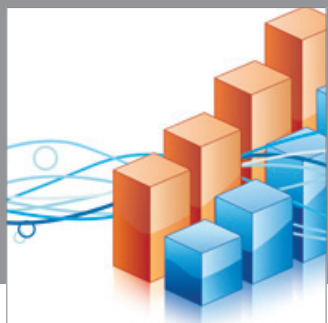

Advances in

Operations Research

mansans

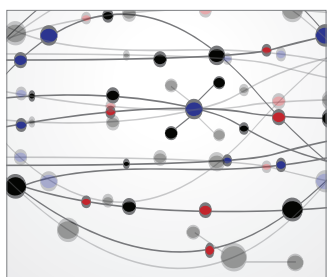

The Scientific World Journal
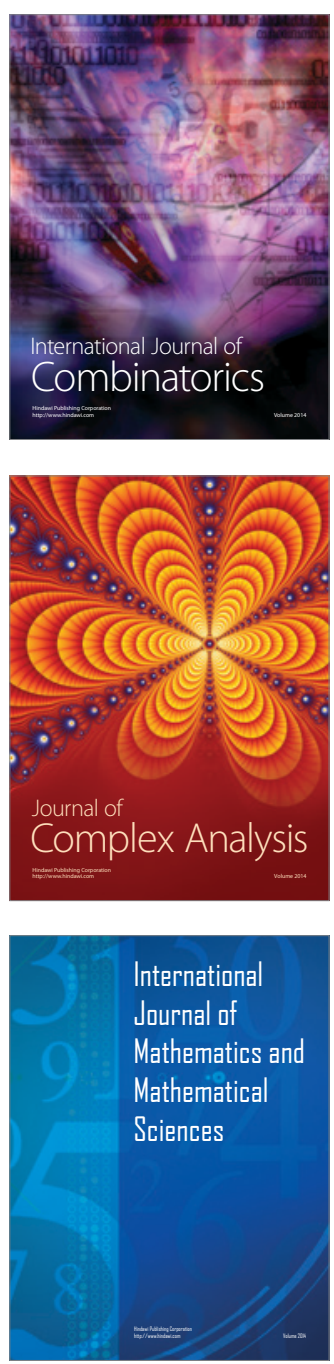
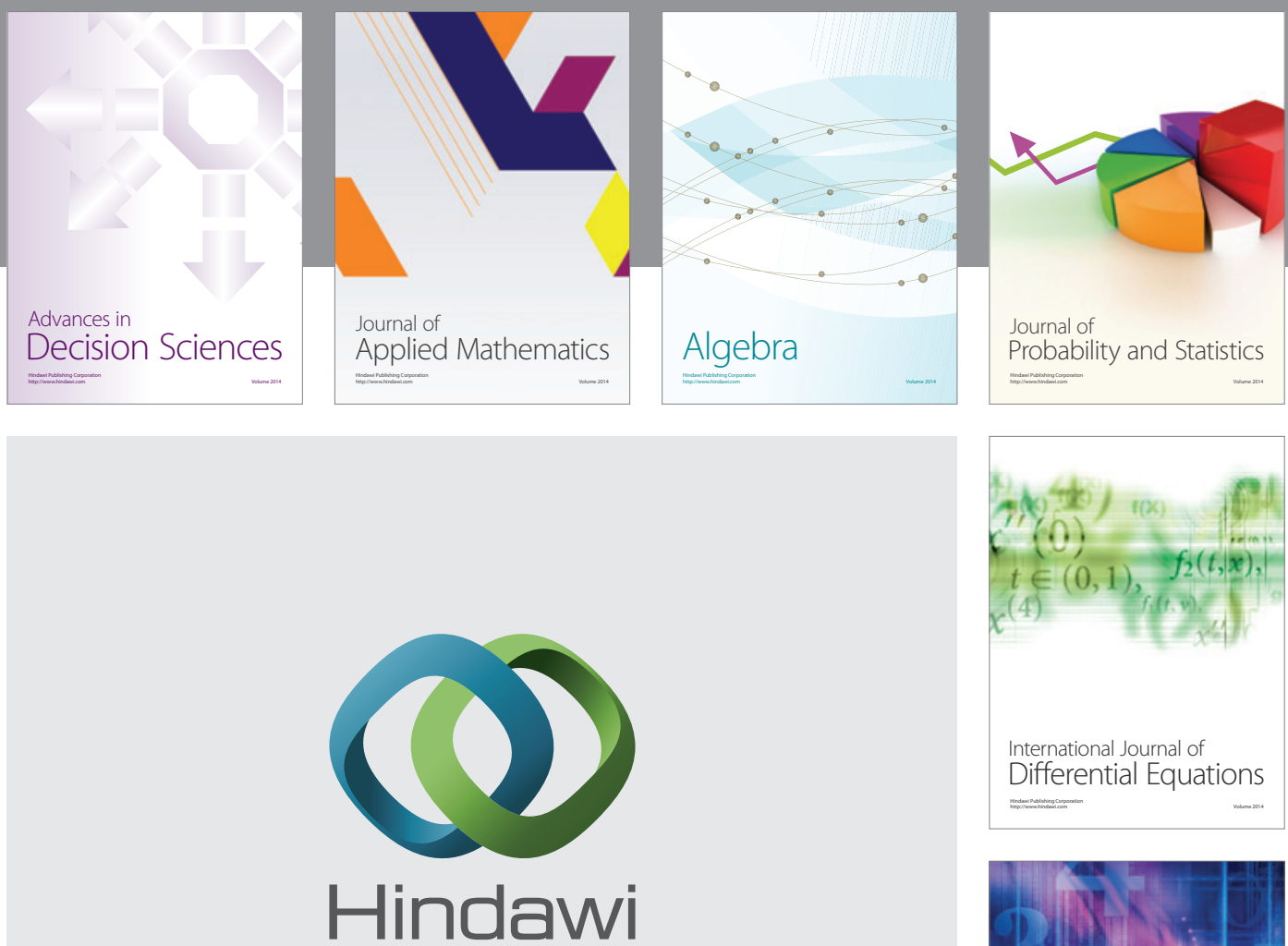

Submit your manuscripts at http://www.hindawi.com
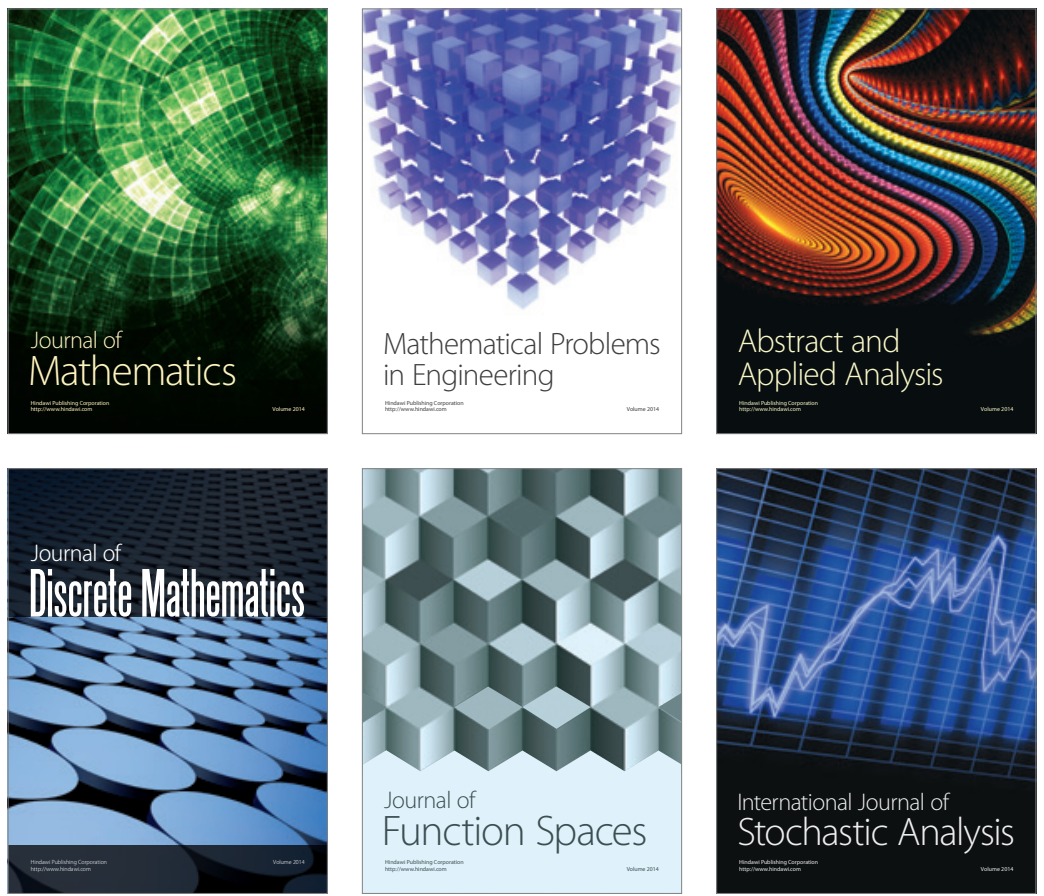

Journal of

Function Spaces

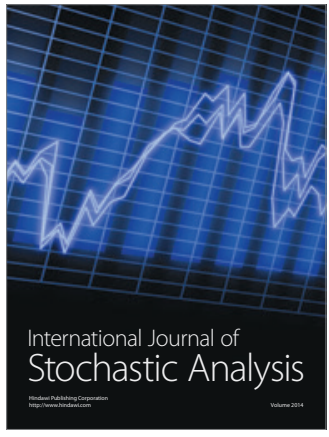

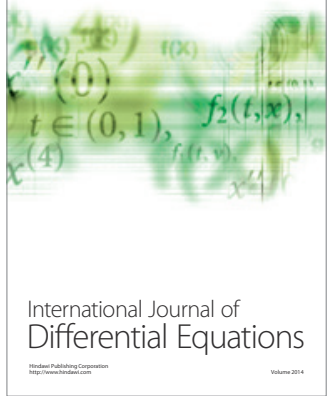
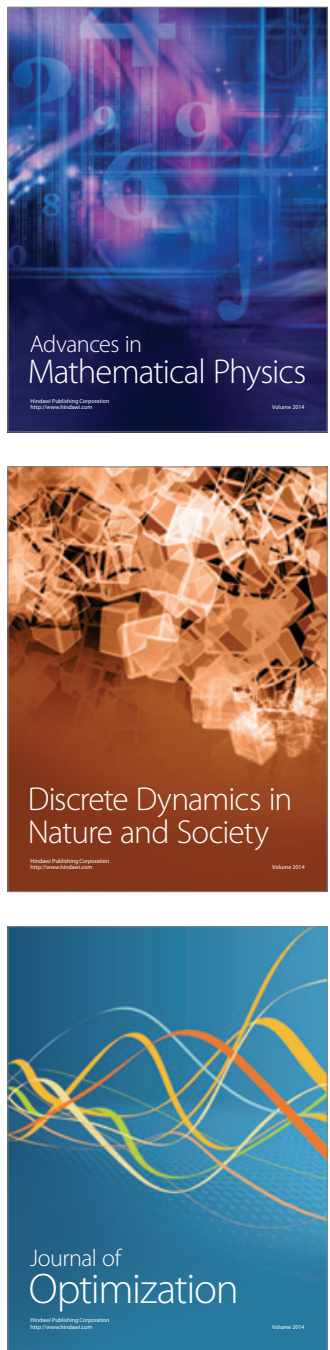